\title{
BMJ Open Understanding the impacts of care farms on health and well-being of disadvantaged populations: a protocol of the Evaluating Community Orders (ECO) pilot study
}

\author{
$\mathrm{H}$ Elsey, ${ }^{1} \mathrm{R}$ Bragg, ${ }^{2} \mathrm{M}$ Elings, ${ }^{3} \mathrm{~J} \mathrm{E}$ Cade, ${ }^{4} \mathrm{C}$ Brennan, ${ }^{1} \mathrm{~T}$ Farragher, ${ }^{1} \mathrm{~S}$ Tubeuf, ${ }^{5}$ \\ R Gold, ${ }^{6}$ D Shickle, ${ }^{1} \mathrm{~N}$ Wickramasekera, ${ }^{5}$ Z Richardson, ${ }^{1} \mathrm{~J}$ Murray ${ }^{1}$
}

To cite: Elsey H, Bragg R, Elings $\mathrm{M}$, et al.

Understanding the impacts of care farms on health and well-being of disadvantaged populations: a protocol of the Evaluating Community Orders (ECO) pilot study. BMJ Open 2014;4:e006536.

doi:10.1136/bmjopen-2014006536

- Prepublication history for this paper is available online. To view these files please visit the journal online (http://dx.doi.org/10.1136/ bmjopen-2014-006536).

Received 9 September 2014 Revised 5 October 2014 Accepted 7 October 2014

CrossMark

For numbered affiliations see end of article.

Correspondence to

H Elsey;

h.elsey@leeds.ac.uk

\section{ABSTRACT}

Introduction: Care farms, where all or part of the farm is used for therapeutic purposes, show much potential for improving the health and well-being of a range of disadvantaged groups. Studies to date have been qualitative or observational, with limited empirical evidence of the effectiveness of care farms in improving health and well-being. Understanding the underlying mechanisms that lead to improvements for different disadvantaged groups is a further gap in the evidence. Participants in this study are offenders serving community orders. Their low socioeconomic status and poor health outcomes relative to the general population exemplifies disadvantage.

Methods and analysis: This paper describes the protocol of a study to understand the impacts of care farms and to pilot the design and tools for a study to assess cost-effectiveness of care farms in improving the quality of life of offenders. As a pilot study, no power calculation has been conducted. However, 150 offenders serving community sentences on care farms and 150 on other probation locations (eg, litter picking, painting) will be recruited over a 1-year period. Changes in quality of life, measured by Clinical Outcome in Routine Evaluation-Outcome Measure, health and reconvictions of offenders at care farms compared to other probation locations will be analysed to inform the sample size calculation for the follow on study. The feasibility of recruitment, retention, collecting cost data and modelling cost-effectiveness will also be assessed. The study will use qualitative methods to explore the experiences of offenders attending care farms and perceptions of probation and care farm staff on the processes and impacts of the intervention.

Ethics and dissemination: Findings will be published and inform development of a natural experiment and will be disseminated to probation services, care farms and academics. University of Leeds Ethical Review Board approved: SoMREC/13/ 014. National Offender Management Service (NOMS) approved: 2013-257.

\section{Strengths and limitations of this study}

- First pilot of a natural experiment to test the cost-effectiveness of care farms in the UK.

- Three different care farm and probation settings are studied using range of methods.

- Probation Services in England are currently undergoing major changes; the timing of this pilot may limit an accurate assessment of feasibility.

\section{BACKGROUND}

Care farming (also called social farming) has been defined as the use of commercial farms and agricultural landscapes as a base for promoting mental and physical health, through normal farming activity. ${ }^{1-3}$ A care farm utilises the whole or part of a farm to provide health, social or educational care services for one or a range of vulnerable groups of people, providing a supervised, structured programme of farming-related activities, rather than occasional one-off visits. ${ }^{4}$ The numbers of care farms have been growing, particularly in Europe, with an estimated 1000 care farms in the Netherlands ${ }^{5}$ and over 230 in the UK, ${ }^{6} 7900$ in France, 300 in Belgium, 160 in Germany, 675 in Italy and 100 in Ireland. ${ }^{8}$

Care farming is a truly complex intervention. Farms differ in terms of the type of farming activities (eg, horticulture and livestock farming), other activities (eg, gardening, conservation, woodwork and metal work) and well-being and skills interventions provided (eg, health promotion, counselling and skills qualifications). There is also a wide range of clients using care farms including those with long-term conditions such as 
dementia, depression, learning disabilities, substance misuse and behavioural issues as well as offenders. Given this complexity the main defining feature of a care farm is the involvement in farm activities for a therapeutic purpose. It is also important to highlight the farming component of the intervention. This helps to distinguish care farms from horticultural or animal-based therapy projects where production is not on a commercial level or as a social enterprise. ${ }^{5}$

Care Farms can be categorised as one element of 'green care'. The typology of green care has been summarised in figure 1 by $\mathrm{Bragg}^{22}$ (adapted from Haubenhofer $e t a l^{9}$; and Sempik and Bragg $\left.{ }^{10}\right)$.

While the number of care farms is increasing across Europe, and their services are increasingly commissioned by a range of public health, education and social sector organisations; commissioners face challenges in identifying the evidence of their effectiveness. The complexities and multifaceted nature of care farms means that this is an intervention that does not lend itself easily to a randomised controlled study design. The observational evidence that is available is published in a wide range of journals or available as 'grey literature' across Europe and is not easily synthesised.

The evidence base for the effectiveness of care farming is relatively recent (within the past 10 years). ${ }^{11}$ Much research originates from the Netherlands and Norway and is comprised of qualitative, cross-sectional and before and after studies with a range of client groups, including the elderly, those with physical or learning disabilities, long-term conditions and psychiatric conditions and with a range of types of care farm.
Findings imply that many participants benefit from; being part of a social community; the relationship with the farmer (and their family and other staff); engaging in meaningful activities in a green environment; and for some, the possibility for work opportunities. ${ }^{12-16}$ The fact that the farm provides an informal, non-care context which is close to the experience of everyday life is also valued. ${ }^{4} 17-19$

Several authors note improvements in mental wellbeing and improvements in social interactions. ${ }^{19-22}$ Positive behavioural impacts such as reduced police contact and drug use have been noted among young people ${ }^{18}$ Reductions in anxiety and depression have been observed in people with mental health issues ${ }^{1415} 192324$ and those with psychiatric and addiction problems have also experienced improved social and work skills. ${ }^{5} 12181921$ 25-28 People with learning difficulties also appear to benefit, with increased life skills and social interaction. ${ }^{21}$ Increased cognitive functioning and wellbeing has been noted among those with dementia. ${ }^{29}$

\section{Why care farms may work}

We hypothesise that the opportunity to not only be in, but also to interact with nature enables care farms to improve quality of life, particularly through improvements in mental health, but also through physical health.

As many care farms also provide opportunities for social interaction, skills building and purposeful work, it is highly likely that these elements also contribute to improved quality of life and well-being. Attempting to unpick these mechanisms for change is challenging and requires further study.

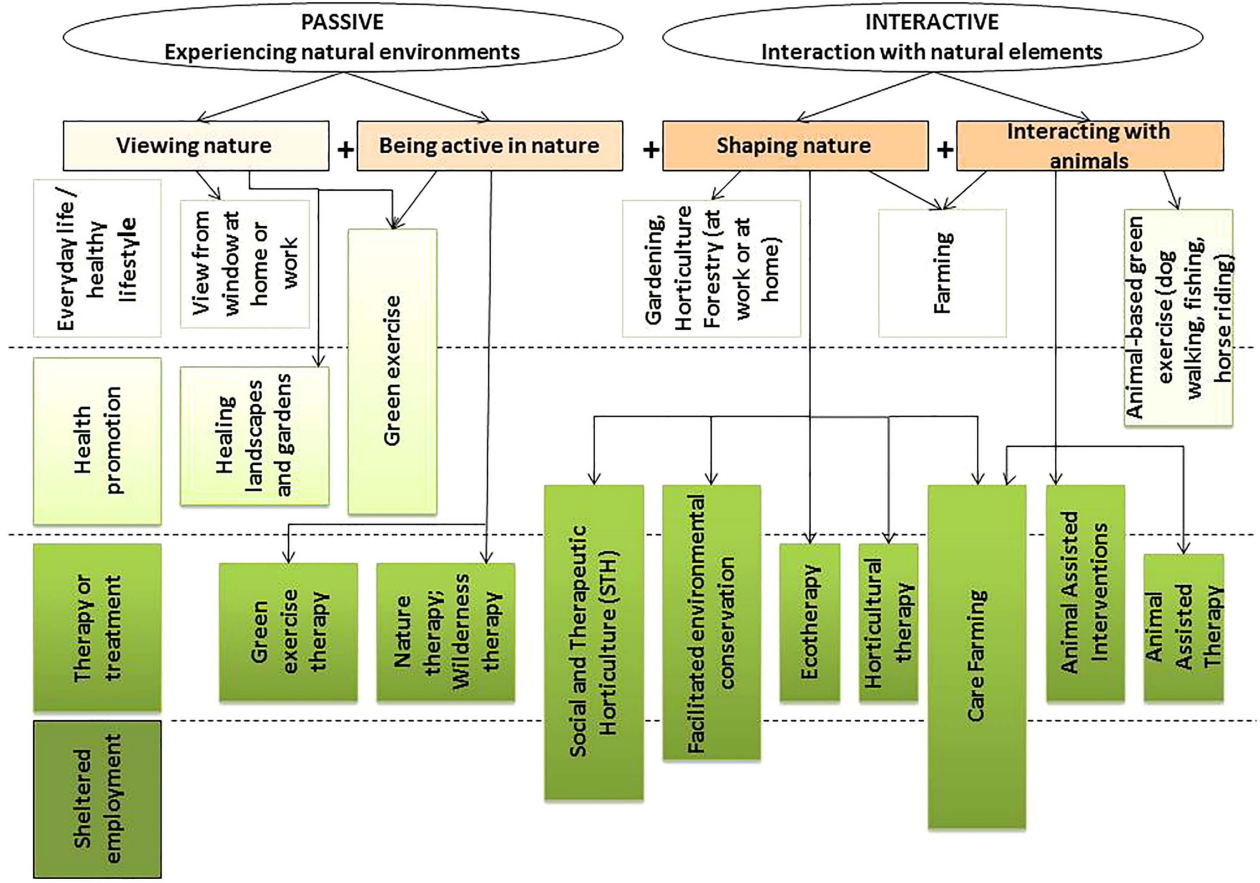

Figure 1 Care farms within the typology of green care. 
Offenders serving probation orders are an important client group for Care Farms in the UK. A survey of 142 care farms in England found 27\% were working with offenders on probation. ${ }^{7}$ While no comprehensive survey of the use of care farms, or social farms across Europe, there are case studies of social farms supporting offenders in Germany and this may well be the case elsewhere in Europe. ${ }^{8}$ A mapping exercise of the use of social/care farms across Europe and potentially further afield would be of value.

Offenders display many of the attributes of a disadvantaged population. They suffer a greater burden of physical and mental ill-health than the general population, ${ }^{30}$ are more likely than the general population to have been in care, ${ }^{31} 32$ suffered harsh or neglectful parenting and developed early behaviour difficulties, ${ }^{31}$ been excluded from school, ${ }^{32} 33$ have witnessed violence at home and suffered from addiction problems as children. ${ }^{34}$ The link between poor mental health and reoffending is well-established. ${ }^{35}{ }^{36}$ The evidence of factors associated with desistance, or not re-offending, highlights the importance of building hope ${ }^{37}$ and social capital, $^{38}$ and changes in perceptions of self ${ }^{37}$ and the interplay of these factors with improvements in opportunities and social, environmental circumstances. ${ }^{39}$ The limited evidence base on green care and care farming would suggest that these environments can produce exactly these sort of benefits and may therefore be particularly appropriate for this and similar client groups.

In England, there is a policy emphasis on the use of community orders, whereby those who have committed lower risk offences are sentenced by the court to serve their punitive order in the community rather than in prison. Community orders have been shown to be more effective than custodial sentences of less than 12 months in reducing reoffending, although this may be in part due to the case mix of offenders. ${ }^{34}$ The positive impacts of community orders have been explained theoretically through concepts of 'generativity' whereby offenders are able to realise personal redemption through positive contributions to the community. ${ }^{40}$ It may be that working on a care farm may also contribute to this sense of generativity.

\section{METHODS/DESIGN \\ Objectives}

The specific objectives of the ECO study are:

1. To conduct a systematic review of published and grey literature evaluating the impacts of care farms in improving the health and well-being of disadvantaged populations.

2. To estimate differences in effectiveness in terms of quality of life, mental health, lifestyle behaviours and reoffending rates between three care farms and between care farms and comparator settings in order to inform sample size calculations for a follow-on natural experiment.
3. To identify factors that drive probation service decisions on where offenders will serve their community order so as to identify potential selection bias and confounders as well as the most appropriate ways to collect data on these factors.

4. To identify the most appropriate ways to gain informed consent, maximise recruitment, follow-up and effective completion of questionnaires while minimising drop out by offenders.

5. To identify the most appropriate ways to collect cost data on the care farm and comparator interventions and wider costs to health and social care and society and explore the feasibility of measuring of conducting cost-utility analysis and/or a cost-benefit analysis.

6. To draw on qualitative work with offenders, care farmers and probation officers to identify the possible mechanisms that lead to changes in quality of life, health and well-being among offenders attending care farms.

\section{Study design: systematic review}

In light of the challenges of synthesising the existing evidence of the effectiveness of care farms in improving health and well-being, a key component of this study is a mixed methods systematic review of published and unpublished evidence (objective 1). The review title has been registered with the Campbell Collaboration and the full protocol will be available on the Campbell Collaboration website. ${ }^{41}$ Details of the review are also available on the PROSPERO website. The study design is summarised briefly here. The aim is to systematically review the available evidence of the effects of care farms on quality of life, health and social well-being of service users. Where possible, the evidence will be synthesised to:

1. Understand the size of the effect that care farms may have on the health, well-being or social outcomes of different population groups.

2. Examine whether effects differ depending on the activities and characteristics of the farm/farmer, the duration of time participants spend at the farm, the number and diversity of the participants on the farm, and whether the farm is the only intervention.

A range of study methodologies including RCTs, nonrandomised observational studies and qualitative studies will be included in the review. As we expect to find some before and after studies that do not have a control group, their inclusion in the review will also be considered. The results of single group pre-post designs will be analysed separately from other study designs. A wider range of population groups use care farms and these will be captured by the review. Participants of any age will be included in the review. We expect the likely participant groups to include: offenders serving community orders or similar sentences in the community rather than in prison; offenders 'on-licence' (ie, recently leaving prison to re-enter the community); people with drug and alcohol problems; people with mental health problems including anxiety, depression and psychiatric 
disorders; young people with challenging behaviour, particularly those excluded/ facing exclusion from school or those at risk of offending; people with health problems particularly long-term conditions, including dementia; people with learning difficulties and people receiving palliative care. It is also possible that the review will identify other relevant client groups.

The primary outcome for the review is quality of life as measured by a validated quality-of-life measure such as the EQ5D, ${ }^{42}$ short-form health survey 36 (SF-36) ${ }^{43}$ CORE-OM ${ }^{44}$ WEMWBS. ${ }^{45}$ This review will cover a broad range of secondary outcomes, including any that use a recognised measure of health, well-being or behaviour, assessed using self-report or objective measures. We aim to use this review and evidence from our primary research to identify pathways to change for different population groups and develop a logic model to explain these relationships. Being too restrictive in the secondary outcomes for the review would limit our understanding of these potential mechanisms.

\section{Study design: pilot study}

The aim of the primary research is to pilot the design and methods of a natural experiment and economic evaluation to examine the effectiveness of care farms in improving the quality of life of offenders serving community orders and to understand the mechanisms within care farms that influence these impacts. The findings of this study will indicate whether a larger, fully powered natural experiment is feasible to assess the cost utility and/or cost-benefit, of care farms in improving offender health and well-being, and ultimately reducing recidivism.

The study is funded by the National Institute for Health Research's (NIHR) Public Health Research Programme and has received ethical approval from the University of Leeds Ethical Review Board (SoMREC/13/ 014) and approval from the National Offender Management Service (NOMS; 2013-257).

The study will use both quantitative and qualitative methods to meet the objectives above. Given the requirement for courts and Probation Services to allocate offenders to locations to serve their community orders, randomisation to care farm or control location would be impossible. Hence, this study explores the feasibility of a natural experiment design whereby offenders attending care farms and comparator locations are assessed at the start and end of their community orders. Qualitative methods will be used to explore processes within probation services, and experiences of offenders and care farm staff.

\section{Target population and setting}

The target population for the study is adult offenders (18 years and over) serving a community order. Offenders who have committed severe offences or have severe mental health issues may occasionally be sentenced to community orders but are not eligible for placement on a care farm, and so will not be included in this study. Resources have been included in the budget for translation services for those who are not comfortable being interviewed in English, thus no one will be excluded based on their ethnicity or language abilities.

In this study, three sites in England will be selected in order to study the variation in Probation Service processes and types of care farms. We will purposively sample Probation Services which have different procedures and structures for working with offenders, including systems for providing initial 'inductions', communication mechanisms and processes for allocating offenders to locations to serve their community orders. We will purposively sample care farms which have a different range of activities both on the farm and also health and support services. For example, some farms offer counselling sessions or health trainers, while others provide skills training in farming or conservation activities or life skills. A few care farms offer qualifications to their clients. Care farms also display a range of organisational cultures, with some working as social enterprises selling the goods that are produced; others have a religious or spiritual focus. There may also be differences in the types of community order that are accepted on different care farms. Some care farms specialise in supporting those with substance misuse problems and may only take offenders with a 'special requirement' for a substance misuse rehabilitation requirement as part of their community order. Understanding these dynamics and how feasible it is to conduct a fully powered study sensitive to these complexities is a key aim of this study.

\section{Comparator locations}

The comparator population will be offenders serving community orders in settings other than a care farm in the same Probation Service areas as the selected farm. The activities carried out while serving community orders in these comparator locations areas may include: building work, working in charity shops, food handling, painting and decorating, recycling and cleaning. Understanding the characteristics of offenders attending the care farms is an important part of establishing the make-up of the comparator arm. Thus, if the participants are allocated to a care farm to serve a special requirement then a group with a similar requirement and risk of re-offending (as determined by the Probation Service assessment process) will be recruited. This understanding of the comparator group will be gained early in the study through discussions with staff in each probation service.

\section{Primary and secondary outcome measures}

The primary outcome of the study is quality of life and well-being derived from the Clinical Outcome in Routine Evaluation-Outcome Measure (CORE-OM). CORE-OM has been validated among offender 
populations $^{46}{ }^{47}$ and can be used to derive QALYs. ${ }^{48}$ The 34 items cover four dimensions: subjective wellbeing; problems/symptoms; life functioning; and risk/ harm. ${ }^{49}$

Secondary outcomes are:

- Individual level data on re-offending rates over a max 18-month period obtained from individual level data from the Police National Computer.

- Mental health derived from Warwick-Edinburgh Mental Well-being Scale (WEMWBS) ${ }^{45}$

- Measures of smoking, alcohol, drug use, diet and physical activity adapted from General Lifestyle Survey ${ }^{50}$ and Health Survey of England. ${ }^{51}$

- Measures of the relatedness to nature..$^{52} 53$

- Exploration of health utility as derived from CORE-OM. ${ }^{49}$ Based on CORE-OM, health states can be valued and quality adjusted life years (QALYs) derived permitting a cost-utility analysis. ${ }^{48}$

- Exploration of the cost per re-offending event avoided due to attendance on a care farm.

\section{Sample and recruitment processes}

As a pilot study, a conventional sample size calculation is not appropriate as the study's main aim is to assess feasibility, recruitment and follow-up rates, clarify selection biases and effects of confounding. As there are no hard and fast rules for judging the sample size for a pilot study, we judge an appropriate sample size to be 300 participants recruited across the three care farms and comparator sites. This will be sufficient to allow us to determine a sample size for a follow-on study that takes account of between-care farm effects and the possible effects of bias (ie, response rates and drop-out). With an expected loss to follow-up of $40 \%$, this will allow a total of 180 participants (90 care farm attendees and 90 comparator location attendees) with both baseline and follow-up data. Using three sites will enable the assessment of variation between care farms and with comparator sites, in terms of: recruitment and follow-up rates, allocation decisions (ie, confounders), selection biases and outcome measures.

In order to meet this target of 300 participants, we plan to recruit 60 participants over a 1-year period from care farm 1 and 60 from comparator 1 . Recruitment will start at a later date in the other two care farms and comparators. Forty-five participants will be recruited from care farm 2 and 45 from comparator 2. Similarly, 45 will be recruited from care farm 3 and comparator 3 . These participants will be recruited over a 9-month period. In total therefore, 150 participants will be recruited from all three care farms and 150 from across the three comparator locations. The delayed start in recruitment in care farms/comparator 2 and 3 will enable us to draw on initial learning on recruitment from care farms/comparator 1 and to try improved strategies in the remaining two sites. Recruitment will be spaced over the entire year in order to identify any impact of seasonality on participants' experience, activities and outcomes. Ideally offenders should be recruited and baseline measures taken prior to start their community order placement. However, this will be dependent on a number of factors including the speed at which placements start after sentencing, and the logistics of integrating research processes within and across multiple probation sites. We will work with probation services and care farm/comparator site staff to establish the most appropriate and feasible time to recruit. The possibility of incentivising offenders to take part in the study will be discussed with probation staff.

Recruitment will be conducted face to face by a research assistant. Informed consent will be obtained to take part in the study and also independently to access personal information from the probation and police services. Participation in the study will not be contingent on granting permission to access personal data.

Assessing feasibility of these recruitment targets, establishing research procedures and identifying the optimal recruitment processes is a key element in this study.

\section{Follow-up}

Measures for offenders attending the care farm and comparator location will be taken at both the start and completion of their community order placement. If the offender has not completed their placement during the 1 year recruitment period, they will be followed up for 6 months from the start of their order, regardless of whether they have completed their order or placement. If an offender does not comply with the requirements of their order and is categorised by the Probation Services as having 'breached', they will be followed up at the end of their subsequent community order or at the end of the follow-up period. If they are given a prison sentence, they will be noted as 'lost to follow up' for the quality of life, health and well-being measures, however their reoffending outcome can be assessed. As a preference, follow-ups will be conducted face to face as close to the end of their placement as possible. However, the unpredictability of community orders, particularly changes to placements and variable completion rates, may necessitate postal follow-up. In these instances a financial incentive to return the questionnaire will be offered to maximise response rates.

\section{Confounders}

While not all confounders are measurable and may not be relevant as they do not introduce bias into the assessment process, the pilot study and systematic review will identify a list of relevant confounders and ways of measuring these. Potential confounding factors at the individual level include: area of residence, employment status, deprivation, age, gender, lifestyle behaviours, health, mental health, social skills and support, ability to engage with others and offending history. Potential confounding factors at the Probation Services level include seasonality, probation staff may also be influenced by their perceptions/knowledge of individual factors above and this 
may in turn influence the allocation to care farm or comparator sites.

As allocation decisions may be based on some of these factors, confounding by indication will need to be addressed in the planned follow on study. This will be carried out through either propensity (probability of being allocated to a care farm) matching, or cases and control, or adjustment by propensity scores in the outcome models. The pilot data will assess feasibility of collecting information on these potential confounders and provide an initial examination of their relevance to the allocation decision by testing the propensity methods.

\section{Analyses}

Feasibility and acceptability outcomes will be reported descriptively. The correlation between CORE-OM and other secondary measure scores for the same person will be estimated from the pilot data. The estimate and its variability of the primary outcome measure will be used in the sample size calculations for the follow-on study. Additionally, the differences in the outcomes between those offenders at care farms and other locations will be estimated from the pilot data. Two potential issues need to be addressed in the statistical analysis. First the outcomes are to be measured at multiple time points, therefore individuals may vary in their number of measurements due to attrition and there is likely to be correlation in an individual's outcomes over time. Second, as the study includes three sites there is potential for clustering of outcomes and other factors for individuals within each site. To account for these issues multilevel models will be used with time points nested within individuals and individuals nested within sites. Using multilevel models therefore accounts for missing data at particular time points, correlation in outcomes for an individual and account for potential clustering between sites. Exploring the pilot data using these approaches provides an estimate of the various relationships to inform the follow-on study analysis plan.

If differences in outcomes are found between care farms, appropriate adjustment in the sample size of the main study will account for the clustering/site effect (ie, the intracluster correlation coefficient (ICC)). The results from studies identified in the literature review will also be drawn on for sample size calculations (including ICC estimation) for the follow-on study, incorporating a sensitivity analysis framework to explore the impact of the variation of estimates from previous studies on the subsequent sample size calculation. ${ }^{54}$

\section{Health economics component}

As this is a pilot study, the economic analysis will be exploratory. The main purpose will be to identify issues, particular in terms of collecting both cost and outcome data. The primary exploratory analysis will be a costeffectiveness analysis, especially a cost-utility analysis, using QALYs as the outcome measure and costs incurred in the provision of the intervention, as well as healthcare resource and social services utilisation as reported by clients. QALYs will be obtained using the CORE-OM data and the mapping algorithm. ${ }^{55}$

The secondary analysis will estimate an exploratory expected incremental cost per re-offending event avoided due to attending a care farm. Drawing on a review of the evidence, we will also explore the suitability of a cost-benefit analysis of care farms to society incorporating reoffending and crime rates and employability of offenders after attending a care farm.

\section{Qualitative sampling and methods}

There are three main areas to the study which necessitate a qualitative approach. First, to understand the factors driving decisions to allocate offenders to care farms or other community order locations, qualitative interviews with approximately three probation staff responsible for making these decisions in each of the three Probation Services will be conducted (objective 3). The team will also explore the possibilities of analysing routine Probation Services data to better understand any systematic differences in the characteristics of those allocated to the care farms as opposed to other community order locations.

The second area to be explored using qualitative methods is the experience of recruitment and conducting the questionnaire. This will meet objective 4; approximately 12 offenders will be sampled from care farm and comparator locations. Half of these will be interviewed immediately following their recruitment and completion of the questionnaire and the remainder will be interviewed immediately after their follow-up questionnaire at the end of their community order. These interviews will explore their understanding of the study, the meaning of informed consent, their perceptions of the research team (whether separate from probation), understanding of the quality of life and relatedness to nature questionnaires, satisfaction with and experiences of the follow-up process, suggestions for improvement.

Third, in response to objective 6 , qualitative in-depth interviews will be used with a purposive sample of offenders attending care farms. A topic guide will be developed based in theories of desistence and green care and will aim to capture their experiences of activities on the farm, impact of weather conditions, any changes their health and well-being and the changes they have experienced during their community order that may have influenced these changes. We will test the feasibility of purposively sampling participants based on change or no change in their quality of life scores. This approach may be challenging as it will require following up participants once their baseline and end-line questionnaires have been analysed and this can only be carried out once their order has ended. This may make them much harder to follow-up. We will use a separate consent process for this part of the study and incentivise participation through the offer of high street vouchers. Six 
in-depth interviews with care farm staff will also be conducted to identify details of activities, support provided, challenges, improvements, their perceptions of the impacts of the care farm on offenders and their articulation of the purpose of the care farm. The researcher will keep a reflective log, paying particular attention to the dynamics and openness of participants during interviews.

\section{Qualitative analysis}

We will be applying a theoretical thematic analysis using theories on desistance and green care to structure the analysis. Theories on desistance suggest a number of factors contribute toward reducing the risk of re-offending including for example building social relationships, offering hope and motivation and developing self-efficacy. ${ }^{37}{ }^{38}$ Green care theories suggest that mental well-being is enhanced through working in nature and interacting with animals. ${ }^{56}{ }^{57}$ We will specifically enquire within the data how these theories might interact within the context of the care farm to understand impacts on attitudes to re-offending and perceptions of well-being. Recorded interviews will be transcribed verbatim by a member of the team who is not involved in the interviewing. Transcripts will be imported into Nvivo for coding purposes. Before coding, each interview transcript will be read and the recording listened to again by the interviewer/analyst with a view to identifying meaningful units of text that relate to theories on desistance and green care and also ensuring accuracy of the transcription process. Coded data will be collated and codes that are repeated across transcripts or appear to be linked will be grouped into initial themes and/or subthemes (the latter may be lower order categories). This stage will involve developing an understanding about the relationships between codes and this may be facilitated by creating maps (MS excel is good for tracking, condensing and sorting data alongside visual maps-coding matrices can be exported from Nvivo into MS Excel). This process will be repeated between interviews allowing us to gauge when saturation has been reached. The number of interviews expressed above should be seen as a guide only and may increase or decrease depending on when saturation can be reached. Although this will be a theoretical thematic analysis we will be open to new potential themes that are not represented by the guiding theories.

\section{Collaboration}

This study is built on the strengths of a multidisciplinary team of researchers with green care expertise from the Essex Sustainability Institute and the Green Exercise Research Team and Plant Research International, Wageningen University; with public health, statistics, qualitative research, health economics and systematic review expertise from the University of Leeds. Another strength of the team is the strong link to Probation Services which has ensured that the question is of relevance to practice. Probation Services users have been engaged in the design of the project and the team will continue to draw on the advice of a service user group facilitated by the Probation Service.

\section{DISCUSSION}

This study will contribute to understanding of the impacts care farms may have on health and well-being and the pathways through which theses impacts are delivered. This study will provide the information needed to design a larger natural experiment to test the cost-effectiveness of care farms in improving the quality of life of offenders. These findings will provide valuable information for policymakers and practitioners seeking interventions for offenders, and may well provide valuable for when considering other disadvantaged groups.

The study is being implemented during a time of significant change within Probation Services in England. The implementation of the UK governments' 'Transforming Rehabilitation' reform program has led to the creation of a new public sector National Probation Service to work with the most high-risk offenders and the establishment of 21 new Community Rehabilitation Companies (CRCs). These CRCs are currently within the public sector, but the sale of shares is imminent. They will manage medium and low-risk offenders. While these organisational changes may necessitate some adaptation and flexibility during study implementation, the timing of the study will allow detailed observation of these changes and their impacts on providers such as care farms.

The combination of primary research and evidence review within this study will facilitate the emergence of holistic findings on the mechanisms through which interactions with nature may influence the health and well-being of disadvantaged populations. This level of understanding has the potential to influence the extent and nature of the provision of green care, adding to the tool-kit of interventions available to lessen health inequities in our societies.

\section{Author affiliations}

${ }^{1}$ Academic Unit of Public Health, University of Leeds, Leeds, UK ${ }^{2}$ Essex Sustainability Institute, University of Essex, Colchester, UK ${ }^{3}$ Plant Research International, Wageningen University, Wageningen, The Netherlands

${ }^{4}$ Nutritional Epidemiology Group, University of Leeds, Leeds, UK ${ }^{5}$ Academic Unit of Health Economics, University of Leeds, Leeds, UK ${ }^{6}$ West Yorkshire Community Rehabilitation Company Limited, Wakefield, UK

Acknowledgements The authors would like to acknowledge the support of the Probation Services' service user group members who have commented on research tools and ideas and advised the team.

Contributors HE, RB, ME, TF, JEC, ST, CB, RG and DS conceived the study and participated in its design. HE drafted and revised the manuscript. JM, NT and ZR implemented the study. All authors commented on the draft.

Funding This project is funded by the National Institute for Health Research's Public Health Research Programme (project number 11/3050/08). The views and opinions expressed therein are those of the authors and do not necessarily reflect those of the Public Health Research Programme, NIHR, the NHS or the Department of Health. 
Competing interests None.

Patient consent Obtained.

Ethics approval University of Leeds Ethical Review Board and National Offender Management Service (NOMS).

Provenance and peer review Not commissioned; internally peer reviewed.

Open Access This is an Open Access article distributed in accordance with the Creative Commons Attribution Non Commercial (CC BY-NC 4.0) license, which permits others to distribute, remix, adapt, build upon this work noncommercially, and license their derivative works on different terms, provided the original work is properly cited and the use is non-commercial. See: http:// creativecommons.org/licenses/by-nc/4.0/

\section{REFERENCES}

1. Hassink J. Combining agricultural production and care for persons with disabilities: a new role of agriculture and farm animals. In: Cirstovao A, Zorini, LO, eds. Farming and rural systems research and extension. Local identities and gloablisation. Florence: Wageningen University/Fifth IFSA European Symposium, 2003:332-41.

2. Hassink J, Van Dijk M, eds. Farming for health. Green-care farming across Europe and the United States of America. Vol 13. Dordrecht Springer, 2006.

3. Hine R, Peacock J, Pretty J. Care farming in the UK: contexts, benefits and links with therapeutic communities. Ther Communities 2008;29:245-60.

4. Bragg R. Care farming in the UK-key facts and figures. Summary report for Natural England. University of Essex, 2013.

5. Elings M, Haubenhofer D, Hassink J, et al. Effecten van zorgboerderijen en andere dagbestedingsprojecten voor mensen met een psychiatrishe of verslavingszorgachtergrond. 2011.

6. Bragg R, Egginton-Metters I, Elsey $\mathrm{H}$, et al. Care farming: defining the 'offer' in England. Natural England Commissioned Reports, NECR, 2014:155.

7. Bragg R, Wood C. Care farming provision and capacity survey 2014 Green Exercise Research Team, University of Essex, 2014.

8. Di Lacovo F, O'Connor D. Supporting policies for social farming in Europe progressing multifunctionality in responsive rural areas, in report for so far: social services in multifunctional farms ('Social Farming'). 2009

9. Haubenhofer DK, Elings M, Hassink J, et al. The development of green care in Western European countries. Explore 2010; 6:106-11.

10. Sempik J, Bragg R. Green care: origins and activities. In: Gallis C, ed. Green care: for human therapy, social innovation, rural economy and education. New York: Nova Science Publishers, 2013.

11. Bragg R. Nature-based interventions for mental wellbeing and sustainable behaviour: the potential for green care in the UK. University of Essex, 2014.

12. Berget B, Skarsaune I, Ekeberg O, et al. Humans with mental disorders working with farm animals: a behavioural study. Occup Ther Ment Health 2007;23:101-17.

13. Kam MCY, Siu AMH. Evaluation of horticultural activity programme for persons with a psychiatric illness. Hong Kong J Occup Ther 2010;20:80-6.

14. Pederson I, Nordaunet T, Martinsen EW, et al. Farm animal-assisted intervention: relationship between work and contact with farm animals and change in depression, anxiety and self-efficacy among persons with clinical depression. Issues Ment Health Nurs 2011;32:493-500.

15. Pederson I, Martinsen EW, Berget B, et al. Farm animal-assisted intervention for people with clinical depression: a randomized controlled trial. Anthrozoos 2012:25:149-60.

16. Elings M. Effects of care farms; scientific research on the benefits of care farms for clients. Trimbos Institute and Practikon/Radbound University: Wageningen UR, 2012.

17. Hassink J, Elings M, Zweekhorst M, et al. Current status and potential of care farms in the Netherlands. Njas-Wageningen $J$ Life Sci 2007;55:21-36.

18. Hassink J, Elings M, Zweekhorst M, et al. Care farms in the Netherlands: attractive empowerment-oriented and strengths based practices in the community. Health Place 2010; 16:423-30.

19. Bragg R, Wood C, Barton J. Ecominds: effects on mental wellbeing. London: Mind, 2013.
20. Hassink J, Meyer R de, Sman P van de, et al. Effectiviteit van ervarend leren op de boerderji. Tijdschrift voor de Orthopedogagiek 2011;50:51-63.

21. Leck C. The impact of care farming in the UK. Worcester: University of Worcester, 2013.

22. Bragg RE. Nature-based interventions for mental wellbeing and sustainable behaviour: the potential for green care in the UK. University of Essex, 2014.

23. Gonzalez MT, Hartig T, Patil GG, et al. Therapeutic horticulture in clinical depression: a prospective study. Res Theory Nurs Pract 2009;23:312-28.

24. Berget B, Braastad BO. Animal-assisted therapy with farm animals for persons with psychiatric disorders. Annali dell'Istituto Superiore di Sanita 2011;47:384-90.

25. Elings $\mathrm{M}$, Hassink J. Green care farms: a safe community between illness or addiction and the wider society. Ther Communities 2008;29:310-22.

26. Berget B, Ekeberg O, Braastad BO. Animal-assisted therapy with farm animals for persons with psychiatric disorders: effects on self-efficacy, coping ability and quality of life, a randomized controlled trial. Clin Pract Epidemiol Ment Health 2008;4:9.

27. Berget B, Ekeberg O, Braastad BO. Attitudes to animal-assisted therapy with farm animals among health staff and farmers. $J$ Psychiatr Ment Health Nurs 2008;15:576-81.

28. Iancu SC, Zweekhorst MB, Veltman DJ, et al. Mental health recovery on care farms and day centres: a qualitative comparative study of users' perspectives. Disabil Rehabil 2013;36:573-83.

29. De Bruin SR, Oosting SJ, Kuin Y, et al. Green care farms promote activity among elderly people with dementia. J Housing Elder 2009;23:368-89.

30. Fazel S, Baillargeon J. The health of prisoners. Lancet 2011;377:956-65

31. Sutton C, Utting D, Farrington D. Support from the start: working with young children and their families to reduce the risks of crime and anti-social behaviour, Report 524. Department for Education and Skills Research, 2004

32. Parke S. Young people in custody 2006-08. HM Inspectorate of Prisons and Youth Justice Board, 2009.

33. Ministry of Justice. Compendium of reoffending statistics and analysis Statistics Bulletin, in Statistics Bulletin. London: Ministry of Justice, 2010

34. Ministry of Justice. Breaking the cycle: effective punishment, rehabilitation and sentencing of offenders: green paper evidence report. 2010.

35. Wallace $C$, Mullen $P$, Burgess $P$. Serious criminal offending and mental disorder: case linkage study. Br J Psychiatry 1998;172:477-84.

36. Fazel S, Yu R. Psychotic disorders and repeat offending: systematic review and meta-analysis. Schizophr Bull 2011;37:800-10.

37. McNeill F, Farrall S, Lightowler C, et al. How and why people stop offending: discovering desistance. Institute for Research and Innovation in Social Services, No. 15. April 2012.

38. Farrall S, Bowling B. Structuration, human development and desistance from crime. Br J Criminol 1999;17:252-67.

39. LeBel TP, Burnett R, Maruna S, et al. The "Chicken and Egg" of subjective and social factors in desistance from crime. Eur $J$ Criminol 2008;5:131-59.

40. McNeill F, Maruna S. Giving up and giving back: desistance, generativity and social work with offenders, In: Mclvor G, Raynor P, eds. Developments in social work with offenders. London: Jessica Kingsley, 2007.

41. Elsey H, Brennan C, Murray J, et al. Do care farms improve the quality of life of disadvantaged populations? Campbell Collaboration, 2014.

42. Szende A, Janssen B, Cabases J. Self-reported population health: an international perspective based on EQ-5D. Springer Link Online: Springer, 2014

43. Ware J, Kosinski M, Bjomer B, et al. User's guide for the SF-36v2 Health Survey. Qualty Metric Incorporated, 2007.

44. Gray P, Mellor-Clark J. CORE: a decade in development. Page Bros, Rugby, 2007.

45. Tennant R, Hiller L, Fishwick R et al. The Warwick-Edinburgh Mental Well-being Scale (WEMWBS): development and UK validation. Health Qual Life Outcomes 2007;5:63.

46. Tapp J, Fellowes E, Wallis N, et al. An evaluation of the Enhanced Thinking Skills (ETS) programme with mentally disordered offenders in a high security hospital. Legal Criminol Psychol 2009;14:201-12.

47. Vallentine V, Tapp J, Dudley A, et al. Psycho-educational group work for detained offender patients: understanding mental illness. J Forensic Psychiatry Psychol 2010;21:393-406. 
48. Brazier J. Measuring and valuing mental health for use in economic evaluation. J Health Serv Res Policy 2008;3:70-5.

49. Gray P, Mellor-Clark J. CORE: a decade of development. Page Bros, Rugby, 2007. http://www.coreims.co.uk

50. Office of National Statistics. General Lifestyle Survey. 2010.

51. NHS Information Centre for Health and Social Care. Health Survey for England. 2012.

52. Nisbet EK, Zelenski JM, Murphy SA. The Nature Relatedness scale: linking individuals' connection with nature to environmental concern and behavior. Environ Behav 2009;41:715-40.

53. Inclusion with nature: the psychology of human-nature relations, in psychology of sustainable development. Springer, 2002:61-78.
54. Roberts $\mathrm{C}$. The implications of variation in outcome between health professionals for the design and analysis of randomized controlled trials. Stat Med 1999;18:2605-15.

55. Mavranezouli I, Brazier JE, Young TA, et al. Using Rasch analysis to form plausible health states amenable to valuation: the development of CORE-6D from a measure of common mental health problems (CORE-OM). Qual Life Res 2011;20:321-33.

56. Hine R, Peacock J, Pretty J. Green spaces: measuring the benefits. Report for the National Trust. National Trust, 2008.

57. Berget B. Animal-assisted therapy: effects on persons with psychiatric disorders working with farm animals. Norwegian University of Life Sciences, 2006. 